\title{
Reproducibility of spirometrically controlled CT lung densitometry in a clinical setting
}

\author{
R.J.S. Lamers*, G.J. Kemerink*, M. Drent**, J.M.A. van Engelshoven*
}

Reproducibility of spirometrically controlled CT lung desitometry in a clinical setting. R.J.S. Lamers, G.J. Kemerink, M. Drent, J.M.A. van Engelshoven. (CERS Journals Ltd 1998. ABSTRACT: The aim of this study was to assess the reproducibility of quantitative, spirometrically gated computed tomographic (CT) lung densitometry at defined levels of inspiration in hospitalized patients.

On two consecutive days, spirometrically gated CT sections were obtained from 20 hospitalized patients at $5 \mathrm{~cm}$ above and $5 \mathrm{~cm}$ below the carina, and at 90 and $10 \%$ of the vital capacity (VC). The mean, modal and median lung densities were calculated, the cut-off points of the frequency distribution of Hounsfield units (HU) defining the lowest and the highest 10th percentile, as well as the histogram full width at half maximum. The lung density parameters of corresponding CT sections of both studies were compared. Reproducibility was expressed as the standard deviation of the signed difference between the results of Day 1 and Day 2 divided by $\sqrt{2}$. Reproducibility data were correlated with results of airflow limitation.

At $90 \% \mathrm{VC}$, reproducibility was of the order of 3-14 HU in both lung zones. At $10 \% \mathrm{VC}$, reproducibility was worse by approximately a factor of three. No relationship was found between reproducibility and results of airflow limitation.

In conclusion, objective measurement of lung density at spirometrically controlled levels of inspiration is a reproducible method in assessing pulmonary density. Reproducibility of lung density measurements is not influenced by severe respiratory insufficiency. The most reproducible computed tomographic lung density measurements can be obtained at $90 \%$ vital capacity.

Eur Respir J 1998; 11: 942-945.
Depts of *Radiology and **Pulmonology, University Hospital Maastricht, The Netherlands.

Correspondence: R.J.S. Lamers

Dept of Radiology

P. Debyelaan 25

University Hospital Maastricht

PO Box 5800

6202 AZ Maastricht

The Netherlands

Fax: 31433876909

Keywords: Computed tomography densitometry

lung

techniques

Received: April 71997

Accepted after revision November 111997
Densitometry of the lung is extensively used in pulmonary research. It has the potential to become an important addition to standard lung function tests in diagnosing and following the extent of pulmonary emphysema and interstitial lung disease. For recent reviews of this extensive work we refer to the literature $[1,2]$. The value of the technique is clearly proven in pulmonary emphysema. During inspiration, computed tomography (CT) correlates well with the pathologic score of emphysema [3]. Expiratory CT reflects airway obstruction and air-trapping more than it does emphysema $[4,5]$. Densitometric data may contribute to improved patient selection and to better evaluation of the response to volume reduction surgery $[6,7]$. Densitometry may provide an additional measure in selecting the side of greatest severity for unilateral lung reduction surgery [7].

The methodologogy of CT densitometry has been established as well as factors affecting parameters derived from a histogram of Hounsfield units (HU). The accuracy and conformity of modern CT scanners have proved to be adequate after correction for poor air calibration $[8,9]$. CT number histograms of the lung are strongly dependent on section thickness and reconstruction filter [10-12]. To optimize CT evaluation procedures, semi-automatic algorithms isolating lung parenchyma by fast contour tracking have been developed, as well as automatic evaluation algorithms calculating various densitometric parameters automatically. Usually, mean lung density is determined as an average over a relatively large area. Unfortunately, it can be spuriously misleading especially at localized disease onset [13]. Mean lung density, however, is not the only densitometric parameter which can be extracted from the CT data. Pixel index analysis $[3,4,14]$ and the lowest 10th percentile of the frequency distribution of densities [15] have shown promising results in the assessment of emphysema.

If densitometry of the lung is used for diagnosis and follow-up of pulmonary emphysema or interstitial lung disease, the overall precision of the procedure has to be established. Reproducibility errors due to instrumental and technical factors have generally been considered to be smaller than patient-related factors. The CT density of the lung is considerably influenced by the level of inspiration at which the CT images are obtained. Control and monitoring this level of inspiration during scanning is of major importance to obtain reproducible CT lung density measurements. A spirometrically controlled CT technique has been developed, offering the opportunity to obtain CT scans at defined levels of inspiration [16].

The purpose of this study was to assess the reproducibility of a number of histogram-related parameters of single CT slices through the upper and the lower zones of the lungs at 90 and $10 \%$ of the vital capacity (VC) in a hospitalized patient population. 


\section{Materials and methods}

\section{Subjects}

Twenty hospitalized patients without the presence of pneumonia or lung tumour on the plain chest radiograph, participated in this study. There were 11 males and nine females, aged 18-63 yrs (median, 44 years). Ten patients had never smoked and 10 were smokers or former smokers (mean consumption was 52 pack-years). Causes of hospitalization were: exacerbation of chronic obstructive pulmonary disease (COPD) $(n=14)$; pulmonary embolism $(n=2)$; haemoptysis $(n=2)$; and chest-wall pain $(n=2)$. The mean VC as measured at the pulmonary function laboratory was $3,830 \pm 1,116 \mathrm{~mL}$ ( $85 \%$ of predicted; range $63-147 \%$ pred), and the mean forced expiratory volume in one second (FEV1) was 2,404 $\pm 1,166 \mathrm{~mL}(82 \%$ pred; range $30-148 \%$ pred). The study was approved by the local Medical Ethics Committee.

\section{Quantitative Assessment of CT scans}

Two CT studies for quantitative assessment of lung density were conducted on two separate days and were performed by two different operators. The topogram was obtained at full inspiration. Two anatomical levels were selected from the topogram: $5 \mathrm{~cm}$ above and $5 \mathrm{~cm}$ below the level of the carina. To control the respiratory status during scanning, the patient was asked to breathe through a small handheld spirometer (Micro Medical Instruments, Rochester, UK), which was connected to the CT scanner [16]. Patients were instructed and allowed 1 min to accustom to the spirometer. Subsequently, the patient performed a breathing VC manoeuvre to determine the VC. At each anatomical level, two scans were obtained at 90 and $10 \%$ of the inspiratory VC, respectively. The airflow through the spirometer was interrupted mechanically by a closing valve, at which time a trigger signal started the scanner. The respiratory status was constant for the duration of the CT scan. The CT scans of the lungs were obtained in supine position with the same CT scanner (Somatom Plus; Siemens, Erlangen, Germany) with $1.0 \mathrm{~mm}$ collimation, $137 \mathrm{kVp}, 220 \mathrm{~mA}$, and $1 \mathrm{~s}$ scanning time. Scans were reconstructed in the soft-detail resolution mode. The full width at half maximum of the point of spread function of this reconstruction filter was $1.86 \mathrm{~mm}$ and the sample volume $3.92 \mathrm{~mm}^{3}$ [12]. No intravenous contrast material was employed. All patients completed the CT study within 10 minutes.

\section{Data analysis}

CT data were digitally transferred for analysis to a Sun SPARCstation (SUN Microsystems, Mountain View, CA, USA). The parenchyma of both lungs was delineated automatically by a density-discriminating computer program, which contoured at a density discriminating level of -200 HU. Subsequently, the contour of the lung was eroded by 3 pixels. Software determined histogram shaperelated parameters automatically: the mean, modal and median lung density; the cut-off points of the frequency distribution of HU numbers defining the lowest 10th and the highest 10th percentile; and the histogram full width at half maximum. The densitometric parameters of the two studies were compared. Reproducibility was expressed in HU as the standard deviation of the signed difference between the results of Day 1 and Day 2 divided by $\sqrt{ } 2$ [17]. This standard deviation may serve as an estimate of the variability of many repeated measurements.

\section{Statistical analysis}

A one-sample t-test was used to determine whether the differences between Day 1 and Day 2 were statistically significant. The Shapiro-Wilk test was used to test if the difference between the data on Day 1 and Day 2 could be considered as normally distributed. Relationships between reproducibility data and $\mathrm{FEV} 1 \%$ pred were evaluated by Spearman's rank correlation test. A probability value less than 0.05 was considered statistically significant.

\section{Results}

The data derived from quantitative analysis of the CT scans are summarized in table 1 and on reproducibility of quantitative CT densitometry measurements expressed in HU in table 2. Similar differences between the various parameters derived from distributions of HU numbers of consecutive CT examinations were observed in the upper and the lower zones of the lungs at $90 \% \mathrm{VC}$. These differences were worse by a factor of three at $10 \%$ VC. At $90 \%$ $\mathrm{VC}$, the relative difference of the mean lung density expressed as a percentage between the first and the second examination was $3 \%$ in the upper as well as the lower zones of the lungs, and 6 and 5\%, respectively, at $10 \%$ $\mathrm{VC}$. The highest 10th percentile of the frequency distribution appeared the most prone to variation at both levels of inspiration.

According to the Shapiro-Wilk tests, the difference data could be considered as normally distributed. No significant difference $(\mathrm{p}<0.05)$ existed between results from any parameter on Day 1 and Day 2, except for the full width at half maximum for the data from $5 \mathrm{~cm}$ below the carina at $10 \%$ VC.

Table 1. - Average densitometric results at 90 and $10 \%$ vital capacity (VC) expressed in Hounsfield units

\begin{tabular}{|c|c|c|}
\hline & $90 \% \mathrm{VC}$ & $10 \% \mathrm{VC}$ \\
\hline \multicolumn{3}{|l|}{ Upper zones } \\
\hline Mean lung density & $-877(23)$ & $-797(53)$ \\
\hline Modal & $-911(23)$ & $-845(54)$ \\
\hline Median & $-900(22)$ & $-825(54)$ \\
\hline Lowest 10 th percentile & $-935(23)$ & $-845(54)$ \\
\hline Highest 10 th percentile & $-809(30)$ & $-691(57)$ \\
\hline Full width at half maximum & $59(15)$ & 76 (13) \\
\hline \multicolumn{3}{|l|}{ Lower zones } \\
\hline Mean lung density & $-865(28)$ & $-779(70)$ \\
\hline Modal & $-914(23)$ & $-847(60)$ \\
\hline Median & $-900(24)$ & $-820(67)$ \\
\hline Lowest 10 th percentile & $-940(23)$ & $-889(57)$ \\
\hline Highest 10 th percentile & $-776(42)$ & $-631(97)$ \\
\hline Full width at half maximum & 64 (14) & $106(26)$ \\
\hline
\end{tabular}

Data are presented as mean, and \$in parenthesis. 
Table 2. - Reproducibility of quantitative computed tomography studies of the lung at 90 and $10 \%$ vital capacity (VC) expressed in Hounsfield units

\begin{tabular}{lcc}
\hline & $90 \% \mathrm{VC}$ & $10 \% \mathrm{VC}$ \\
\hline Upper zones & & \\
Mean lung density & 3 & 10 \\
Modal & 6 & 13 \\
Median & 4 & 13 \\
Lowest 10th percentile & 5 & 17 \\
Highest 10th percentile & 9 & 31 \\
Full width at half maximum & 6 & 9 \\
Lower zones & & \\
Mean lung density & 3 & 11 \\
Modal & 6 & 20 \\
Median & 5 & 15 \\
Lowest 10th percentile & 7 & 18 \\
Highest 10th percentile & 14 & 38 \\
Full width at half maximum & 7 & 12 \\
\hline
\end{tabular}

No statistically significant relationship was found between reproducibility of quantitative $\mathrm{CT}$ densitometry and FEV $1 \%$ pred.

\section{Discussion}

The reproducibility of spirometrically controlled CT densitometry of the lung was surprisingly good. The most reproducible CT lung density measurements were obtained at $90 \%$ VC. Reproducibility was worse by a factor of three at $10 \%$ VC. In the upper and the lower zones of the lungs, comparable differences in measurements between two successive scans were observed. The highest 10th percentile of the frequency distribution appeared to be more prone to variation. From $90 \%$ VC to $10 \% \mathrm{VC}$, the mean lung density increased by $80 \mathrm{HU}$ in the upper zones of the lungs and $86 \mathrm{HU}$ in the lower zones. This is much less than reported by WHB et al. [18], who found an increase in attenuation of $200 \mathrm{HU}$ in healthy volunteers. Our numbers are more consistent with air-trapping and are a reflection of the study population, in which patients with COPD are well represented.

Lung density is determined by the relative proportions of gas, blood, extracellular fluid and pulmonary tissue. Computerized methods using standard techniques calculate the density characteristics automatically from the frequency distribution of HU numbers. The highest 10th percentile, the full width at half maximum, the modal lung density, and the median lung density have at present no proven clinical relevance in the quantification of pulmonary emphysema or interstitial lung disease. However, lung density parameters were chosen because they reflect different aspects of the shape of the histogram of CT numbers. The highest 10th percentile of the frequency distribution was the densitometric parameter that showed the greatest variability between successive scans. This difference is probably caused by the contour tracing algorithm used for isolating the lung. This algorithm is inspiration dependent, which possibly results in a different cut-off point of central vessels.

The degree of lung inflation as measured by direct spirometry has been shown to vary markedly with identical breathholding instruction on a series of consecutive CT scans [19]. In co-operative patients, breathholding at full inspiration is considered the most reproducible lung volume, resulting in the lowest variation in lung density between consecutive scans [19]. Submaximal efforts have major effects on lung density measurements, as density values vary inversely with lung volume. Monitoring of the patient's effort will minimize patient-related errors and can be accomplished by spirometric gating of the CT scanner [16]. The flow-volume curve appears on the display monitor of the CT scanner. Feedback control of the spirometric curve makes it is easier to coach patients for a truly maximal effort, especially in those who suffer from severe airflow limitation, and it is of indispensable help to assess patient discomfort. Submaximal effort is easily detected and the scan can then be cancelled. Partly for that reason, reproducibility was not influenced by very severe respiratory insufficiency. Moreover, the range of mean attenuation values from inspiration to expiration is narrower in patients with COPD than in patients with normal FEV $1 \%$ pred values [20].

The aim of the study was to address the question of respiration control on the reproducibility of standardized CT lung density measurements. Since maximal efforts with spirometrically controlled CT in supine position may be particularly fatiguing for dyspnoeic patients, we decided to scan at 90 and $10 \%$ of the VC. If reproducibility of histogram-related parameters is satisfactory at both levels of inspiration, a free choice on grounds of clinical interest can be made. Incidentally, the densitometric procedure had to be aborted because a patient was unable to reach the $90 \% \mathrm{VC}$ or the $10 \% \mathrm{VC}$ trigger level at the end of the procedure. After a timely interruption of the spirometric procedure, density measurements could always be completed successfully after $1 \mathrm{~min}$ of rest. We finished the complete spirometric procedure including the VC manoeuvre within about $10 \mathrm{~min}$.

Noninvasive measurements of lung density by quantitative CT have a great potential for diagnostic purposes and follow-up after therapy. Reproducibility aspects have to be taken into account before application of this technique in practice. At $50 \% \mathrm{VC}$, the standard deviation of the estimat-ed mean attenuation values was after a 5 minute pause $10 \mathrm{HU}$ [13]. This level of inspiration is suitable to differentiate between patients suffering from idiopathic lung fibrosis and patients with COPD. However, this level is of limited value in the differentiation between COPD, pulmonary emphysema and healthy parenchyma. CT scans obtained at full expiration have been shown to correlate well with physiological phenomena consistent with airflow limitation $[4,5]$. Small airways disease may remain completely undetected on routine suspended full inspiration scans and become conspicuous only at suspended full expiration. Paired inspiratory-expiratory CT scans are useful for distinguishing small airways disease from healthy lungs and pulmonary emphysema [20].

There are a few potential sources of variation in the level of inspiration which could influence the reproducibility of CT density measurements. Differences between the two operators in patient coaching may have an important impact on the VC manoeuvre, the result of which is subsequently used for gating the CT scanner. Patients are more familiar with the spirometer on the second day. Although VC shows variation over the day within individuals [21], examinations were arbitrarily scheduled without taking the point of the day into account, to reflect the 
clinical situation as closely as possible. In patients with COPD, bronchodilatator therapy was continued between CT scans. No differences in the clinical state of the patients were reported. Differences between the data on Day 1 and Day 2 were normally distributed and no statistically significant difference was noted between the data from Day 1 and Day 2, which makes a systematic source of variation in VC measurements unlikely.

In conclusion, results of quantitative, spirometrically controlled computed tomographic densitometry are highly repeatable estimates of lung density. Reproducibility of lung density measurements was not influenced by severe respiratory insufficiency. In expiration, reproducibility was worse by a factor of three. If computed tomographic scans obtained at residual volume are used for diagnosis and follow-up, respiratory control is mandatory.

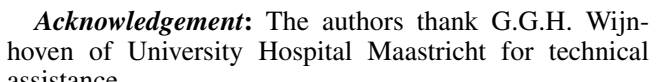
assistance.

\section{References}

1. Morgan MDL. Detection and quantification of pulmonary emphysema by computed tomography: a window of opportunity. Thorax 1992; 47: 1001-1004.

2. Gevenois PA, Yernault JC. Can computed tomography quantify pulmonary emphysema? Eur Respir J 1995; 5: 843-848.

3. Gevenois PA, de Maertelaer V, De Vuyst P, Zanen J, Yernault JC. Comparison of computed density and macroscopic morphometry in pulmonary emphysema. Am Rev Respir Dis 1995; 152: 653-657.

4. Gevenois PA, De Vuyst P, Sy M, et al. Pulmonary emphysema: quantitative CT during expiration. Radiology 1996; 199: 825-829.

5. Gould GA, Redpath AT, Ryan M, et al. Lung CT density correlates with measurements of airflow limitation and the diffusing capacity. Eur Respir J 1991; 4: 141-146.

6. Bae KT, Slone RM, Gierada DS, Yusen RD, Cooper JD. Patients with emphysema: quantitative CT analysis before and after lung volume reduction surgery. Radiology 1997; 203: 705-714.

7. Holbert JM, Brown ML, Sciurba FC, et al. Changes in lung volume and volume of emphysema after unilateral lung reduction surgery: analysis with CT lung densitometry. Radiology 1996; 201: 793-797.
8. Kemerink GJ, Lamers RJS, Thelissen GRP, van Engelshoven JMA. Scanner conformity in CT densitometry of the lungs. Radiology 1995; 197: 749-752.

9. Kemerink GJ, Lamers RJS, Thelissen GRP, van Engelshoven JMA. CT densitometry of the lungs: scanner performance. J Comput Assist Tomogr 1996; 20: 24-33.

10. Kemerink GJ, Kruize HH, Lamers RJS. Density resolution in quantitative computed tomography of foam and lung. Med Phys 1996; 23: 1697-1708.

11. Kemerink GJ, Kruize HH, Lamers RJS, van Engelshoven JMA. CT lung densitometry: dependence of CT number histograms on sample volume and consequences for scan protocol comparability. J Comput Assist Tomogr 1997; 21: 948-954.

12. Kemerink GJ, Kruize HH, Lamers RJS. The CTs sample volume as an approximate, instrumental measure for density resolution in densitometry of the lungs. Med Phys 1997; 24: 1615-1620.

13. Kohz P, Stäbler A, Beinert T, et al. Reproducibility of quantitative, spirometrically-controlled CT. Radiology 1995; 197: 539-542.

14. Müller NL, Staples CA, Miller RR, Abboud RT. "Density mask": an objective method to quantitate emphysema using computed tomography. Chest 1988; 94: 782-787.

15. Gould GA, MacNee W, McLean A, et al. CT measurements of lung density in life can quantitate distal airspace enlargement: an essential defining feature of human emphysema. Am Rev Respir Dis 1988; 137: 380-392.

16. Kalender WA, Rienmüller R, Seissler W, Behr J, Welke M, Fichte H. Measurement of pulmonary parenchymal attenuation: use of spirometric gating with quantitative CT. Radiology 1990; 175: 265-268.

17. van Hastenberg RPJM, Kemerink GJ, Hasman A. On the generation of short-axis and radial long-axis slices in thallium-201 myocardial perfusion single-photon emission tomography. Eur J Nucl Med 1996; 23: 924-931.

18. Webb RW, Stern EJ, Kanth N, Gamsu G. Dynamic pulmonary CT: findings in healthy adult men. Radiology 1993; 186: 117-124.

19. Robinson P, Kreel L. Pulmonary tissue attenuation with computed tomography: comparison of inspiratory and expiratory scans. J Comput Assist Tomogr 1979; 3: 740-748.

20. Lamers RJS, Thelissen GR, Kessels AG, Vlouters EF, van Engelshoven JM. Chronic obstructive pulmonary disease: evaluation with spirometrically controlled CT lung densitometry. Radiology 1994; 1993: 109-113.

21. American Thoracic Society. Lung function testing: selection of reference values and interpretative strategies. Am Rev Respir Dis 1991; 144: 1202-1218. 